\title{
The Atheistic Argument from Outrageousness
}

\author{
Bryan Frances
}

Forthcoming in Think

\begin{abstract}
:
When pressed, many atheists offer three reasons why they reject theism: there is strong evidence against theism, there is no strong evidence for theism, and theism is so outrageous that it needs a great deal of support in order for us to believe it in a reasonable manner. I examine the third reason, arguing that it fails.
\end{abstract}

One reason so many philosophers and other intellectuals are atheists has nothing to do with the alleged weaknesses in the evidence for theism. Neither has it anything to do with the alleged strength of the evidence against theism. Instead, it has to do with the purported outrageous nature of theism.

Theism looks simply incredible relative to our unproblematic knowledge about the world. One hears remarks to this effect all the time-even from theists. I once heard a prominent theistic philosopher of religion joke that he has this "invisible friend" who he talks to; I once heard another say, before beginning a talk that was premised on the truth of theism, that he realized that many audience members will take that premise to be about as plausible as the idea that the Easter Bunny really exists.

Theism is about as outlandish as the idea that the M oon is made of cheese. The proposition that the Moon is made of cheese could be made non-outlandish. Suppose we found out that Venus is made of chocolate, Mars is made of frozen yoghurt, and Jupiter is nothing more than a huge bag of onions smashed together. Well, then the M oon-cheese proposition would no longer be incredible. We still would have no evidence for the Moon-cheese proposition, but we could no longer say it's beyond the pale. Similarly, if we discovered an invisible friend who was omniscient but not omnipotent, or a visible friend who could create whole clusters of galaxies with just thought, then perhaps theism would no longer be incredible. But we have found nothing even approximately like theism to be true: we have no unproblematic knowledge of anything remotely like an omniscient, omnipotent, and wholly good and benevolent creator. Or so many atheists think.

When a theist engages an atheist in debate, they will inevitably cover some of the arguments for and against theism. If the theist is a real expert, she will be able to cast wholly reasonable doubt on a good portion of the alleged defects in pro-theism arguments. She will also, let us assume, be able to reveal important defects in the arguments against theism. But at that point the atheist typically doesn't give up. Instead, he will say 'Yeah, but theism is just so damn crazy compared to what we already know!'

That's the Argument from Outrageousness, AFO. I will argue that it's no good. 


\section{The Weirdness of Science}

M ost of us tend to think that over the last few centuries science has provided a big objection to religion: if science discloses truth, then many of the reasons for thinking God really exists fall apart. I think that's

right, but in another way science supplies an indirect argument against the AFO, as I'Il try to show in this section.

Science has proven that reality is almost unimaginably bizarre. Just think about what we know about the universe. The earth is pretty damn big: about 25,000 miles all the way around. But when you compare the size of the earth to that of our solar system, it's like comparing a grain of sand to the whole beachthat's how comparatively minuscule our earth is. The same holds when comparing our solar system to our galaxy: just a grain of sand. And our galaxy? When it comes to the cosmos our enormous galaxy is just a grain of sand compared to a beach. Finally, some physicists have speculated-but it's only speculation at this point-that there are zillions of universes out there, completely cut off from one another, so each universe is like a self-contained soap bubble. So you are a grain of sand on earth, which is a grain of sand in the solar system, which is a grain of sand in our galaxy, which is a grain of sand in our cluster of galaxies, which is a grain of sand in our universe, which is a grain of sand in all of reality.

Now consider Einstein's theory of relativity. Suppose two things happen: you turn on the oven, call that event $X$, and some person honks the horn in her car, call that event $Y$. It's seems as obvious as anything ever gets that exactly one of the following has got to be true: either $X$ happened before $Y, Y$ happened before $X$, or they happened at the same time. Surely that's obvious, right? But no: according to the standard interpretation of the theory of relativity all three options are true. That is, from some physical perspectives, $X$ happened before $Y$; from others, $Y$ happened before $X$; and from yet others $X$ and $Y$ happened at the same time. None of the perspectives is the "right" one. Whether $X$ happened before $Y$ is relative to the physical perspective in question, on how people in the three perspectives would be moving relative to $X$ and $Y$. How $X$ and $Y$ are temporally related to one another is perfectly objective in the sense that it doesn't depend on what any person thinks or feels, but it does depend on the physical perspective from which $\mathrm{X}$ and $\mathrm{Y}$ are seen. Or so many physicists believe.

Now consider quantum mechanics, which is the mathematics behind the theory of atoms, electrons, protons, and other microscopic particles. On the one hand, this is probably the most impressive theory ever developed. For instance, its predictive accuracy is simply mind-boggling (e.g., some of its calculations are accurate to about one part in a trillion). But there is a version of quantum mechanics, due to the work of physicist David Bohm, which says that the entire universe consists of just one particle that exists in a physical space of almost unlimited dimensions. The activity of that one particle generates the entire universe, including people. No one knows whether Bohm's one-particle view is true, but it's taken seriously as a live option. It's hard to imagine anything weirder than that.

Or consider mathematics. Suppose you have a collection of objects $01,02,03$, etc. Now you add an object to that collection-an object that was not already in the collection and one that really and truly exists. You would think that the collection is bigger now: it has increased in size because it has one more thing in it. But no! Mathematicians insist that if the original set was infinite in size (e.g., maybe it was the 
collection of all even numbers) then adding a new thing to it doesn't make it any bigger: it was infinite to start with and after adding one new item its size is still infinite: it's the same size even though you added something new to it.

That might not strike you as too weird, at least if you think about it for a while. After all, when I said that you had a collection of objects and you add a new item to it, you were probably implicitly thinking of a finite set, such as the set of letters of the English alphabet, and not an infinite set. You may have thought to yourself something like this:

Well, sure! If you've got a collection that has infinitely many things in it, and then you add one more, you're still going to have infinitely many things in it. Infinity plus one equals infinity. Big deal! There's nothing bigger than infinity, so you can't really make the collection any bigger.

That's entirely reasonable. But there are two problems with it.

First, it suggests that the number of even numbers $2,4,6$, 8, etc. is the same as the number of even and odd numbers 1, 2, 3, 4, etc. Both collections of numbers have the same number of members: infinity. Most people have difficulty accepting that.

Second and more importantly, the idea that you can't make an infinite set any bigger is wrong too, because there are lots of different infinities: some infinities are bigger than others. I mean: you can have a collection that has infinitely many things, add some new things to it, and end up with a bigger collection-a bigger infinity. It won't do any good to add just one thing, or even 99 trillion, but if you add the right kind of new infinite collection to the old infinite collection, then you get a bigger infinite collection. For instance there are more numbers from 0 to 1 (including all the decimals) than there counting numbers, $1,2,3$, 4, etc. Weird but true.

Or consider biology. It really is hard to reflect on the human eye, or the heart, or photosynthesis, and think anything other than 'How on earth did nature get this way?' The idea that atoms and molecules randomly moving through space subject to blind forces like gravity and electromagnetism could create eyes and hearts seems crazy. Instead, it certainly looks as though someone had to have designed the human eye and the other amazing parts of nature. So, we have a variant of the traditional design argument for the existence of God-which philosophers know is no damn good. But when we examine nature we do learn that biological life is incredible: over millions of years of random fluctuations things like eyes and hearts can come about naturally just by changing in accord with the laws of nature. Now, some theists will say that God designed the laws of nature. I don't know of any good evidence for that view, but my point here is just this: microscopic particles zooming around obeying the laws of nature naturally produced all the wonderful biological things on earth, and that is just incredible no matter how the laws of nature came about.

Here's another completely amazing thing: did the universe have a beginning, with the Big Bang perhaps, or did it always exist? There are just three possibilities, and each one is utterly bizarre: 
a. The universe had no beginning, so it goes back in time infinitely. So the question 'Where did matter come from?' has no real answer.

b. The universe had a beginning, but nothing caused it to start up. So the birth of matter had no cause whatsoever. It just started up without anything at all making it happen.

c. The universe had a beginning, and something - call it X-caused it to happen. So X would have to be non-physical, since it caused the beginning of the physical universe: $X$ is a non-physical thing causing the universe to happen.

Pretty strange, no? Yet consider this: exactly one of (a)-(c) has simply got to be true (as those are the only possibilities) and yet each one is mind-boggling.

And there are other, related questions. For instance, was Einstein really right about spacetime, thinking of it as a single entity with bizarre properties?

I could go on, with more amazing things about our universe, but why bother? It isn't hard to realize that the universe is utterly astonishing in the sense that it contains things and features that were totally unpredictable from the standpoint of scientifically uninformed common sense.

I am not saying that any of this weirdness provides decent evidence for theism. But when atheists think to themselves that theism is utterly bizarre, they should remind themselves that that's not a good reason to think it's unlikely to be true. The universe is filled with the bizarre, so it would be foolish of us to reject theism just because it's bizarre.

\section{The Weirdness of Philosophy}

Philosophy is still going as strong as ever in $21^{\text {st }}$ century, and part of the reason is that over the millennia people have uncovered mysteries that have resisted solution and show that our universe is astonishing. I'll go over a few of those mysteries here.

A good portion of these mysteries have to do with us as human beings. Is consciousness just a physically generated aspect of the brain? And if, as seems likely, consciousness is physically generated, how does that happen: how does the technicolor of consciousness arise from the electrochemical activity of the grey matter of our brains? No one knows.

Another mystery, about meaning: do our lives have any overarching meaning or purpose, or are meaning and purpose things we invent for ourselves as we go along? Ethics supplies eternal questions as well: are there any real moral truths, or does morality reflect nothing but a bundle of subjective preferences and emotions that we are genetically programmed to accept or are indoctrinated into? Is free will ruled out by the (alleged) fact that we are just highly complex biological systems wholly obedient to stubbornly impersonal physical laws of nature-exactly like any other flesh and blood organism? No one knows. 
All of those are familiar philosophical mysteries, ones you can find in many popular philosophy books and standard introductory courses in philosophy. In saying that they are "familiar" I don't mean to say that they are easy in any way: clearly, some of them are profoundly difficult to probe. I mean rather to point out that those mysteries are well-known in that many educated people outside of universities are aware of at least some of them.

So what other philosophical mysteries are there, ones completely out of public view?

One unexpected mystery suggests-and I kid you not-that there are no ordinary material objects such as cats or cups. You think you have a can and a coffee cup? Nope, not really. There are electrons and other particles where you think your cup and car are, but there is no cup or cat there. The universe is nothing more than a swarm of particles; electrons exist but cats, cups, and humans do not.

Another mystery suggests that the whole notion of linguistic and cognitive meaning is an illusion: so 'I have shoes on' and 'Bill Clinton was President of the USA' are meaningless, as is every sentence and word in this essay and every thought running through your mind. The arguments for this astonishing thesis have been analyzed for centuries, and there is no significant agreement as to what's wrong with them.

Yet another mystery seems to show that the notion of truth is contradictory in the sense that it is simply incoherent: nothing is true and nothing is false-including this very statement. You probably think that we know of tons of truths, such as 'I have clothes on', 'Twice two is four', and 'M iles Davis had soul'. But even if the mystery of meaning (in the previous paragraph) is illusory, another mystery says that no statement whatsoever is true because the notion of truth is as contradictory as the notion of a naked woman with a dress on.

The final mystery appears to show that even if there are trees and tables, none of them are green, brown, or any other color. The universe is utterly colorless. There may be bananas, but none of them are yellow. Oh, and one of the consequences of our colorless universe might be that our minds are partially non-physical, although there is no hope here for an afterlife (not to say there's no hope elsewhere).

Most philosophers do not accept these radical views. They think that there must be some flaw in the arguments for such insane thoughts. The problem with their view (a problem they are fully aware of) is that no one has been able to convince others that he or she has put their finger on the flaws in the arguments for those stunning conclusions. And this remains true despite many centuries of investigation into the arguments by a good portion of the best and brightest minds that have ever walked on our planet.

As was the case with scientific weirdness, there is no argument here for theism. Instead, what we have is an argument that reality is incredibly strange-so strange, in fact, that the oddness of theism is not good evidence that it's false. This point is trickier than it looks, so I will elaborate. 
It's pretty reasonable to get excited about the success of science. There are zillions of things that our ancestors were completely clueless about that we have figured out. What is the sun, why does it come back every day, and why is it hot? How do things grow? What makes babies grow inside a woman? Why are there the seasons? How come rain can become snow? These and many other questions were completely beyond our ancestors.

We know the answers now. In fact, science is putting together a comprehensive view of reality, one that gets tested for accuracy a million times a day in all sorts of ways. If science's picture was wrong, there is no way medicine would work, I-phones would work, electricity would work, airplanes would work, etc. Furthermore, and this point is crucial, there is nothing in this view that looks anything like theism. Science hasn't discovered a bunch of non-physical objects interacting with physical objects, as standard versions of theism require. Science hasn't discovered any super amazing beings who are anything close to omnipotent or omniscient. Science hasn't discovered anyone turning water into wine or raising the dead. Science hasn't discovered anything like heaven or hell or any other kind of afterlife.

In sum: science is enormously successful and hasn't found anything remotely like theism. Given all that, theism seems really improbable. Or so an intelligent person might think. That's the AFO.

But the things I went over in these two "weird" sections (science and philosophy) show that our universe is still incredibly mysterious and unknown to us. There is little reason to think "we have things figured out now": we most definitely do not have things figured out now despite the fact that we are a million times more knowledgeable than our ancestors. More to the point, even if theism is utterly bizarre compared to what our incredibly successful science has shown, this doesn't amount to a good argument that theism is improbable, because that same science-as well as philosophy-has shown that reality is filled with the utterly bizarre. So the AFO just doesn't amount to a compelling argument. 